\title{
Propuesta de una arquitectura para la gestión de información personal en entornos móviles
}

Proposal of an architecture for the management of personal information in mobile environments

Propôs uma arquitetura para o gerenciamento de informações pessoais em ambientes móveis

DOI: http://dx.doi.org/10.23913/reci.v6i11.63

Elizabeth Moreno Galván Instituto Politécnico Nacional. Escuela Superior de Cómputo (ESCOM-IPN), México eingelymg@hotmail.com

Elena Fabiola Ruiz Ledesma Instituto Politécnico Nacional. Escuela Superior de Cómputo (ESCOM-IPN), México efruiz@ipn.mx

Chadwick Carreto Arellano Instituto Politécnico Nacional. Escuela Superior de Cómputo (ESCOM-IPN), México ccarrectoa@ipn.mx

\section{Resumen}

La información juega un papel primordial para la toma de decisiones en las organizaciones e inclusive la vida cotidiana. Actualmente, personas y organizaciones disponen de sus datos en forma física o digital, siendo administrados mediante diferentes protocolos, aplicaciones y sistemas como bancos, nóminas, redes sociales y bases de datos por mencionar algunos; en todos los casos se cuenta con algún nivel de protección y confidencialidad para asegurar la integridad y coherencia de la información, además de la existencia de arquitecturas orientadas a la comunicación y compartición de datos entre sistemas. Sin embargo, aún no 
se logra una interoperabilidad total entre los mismos de forma que sea posible la compartición de información de forma segura y transparente a los usuarios.

En el presente artículo los objetivos serán: 1) Realizar un análisis de las arquitecturas existentes, 2) describir el proceso de diseño de una arquitectura que se propone para la gestión de información personal y, 3) determinar las funcionalidades de cada etapa. La investigación es de carácter descriptivo de acuerdo a los objetivos planteados y para la construcción de la arquitectura se siguieron los pasos del método científico.

Los resultados obtenidos se resumen en la generación de una arquitectura que se presenta como una guía para el desarrollo e integración de sistemas dedicados a la gestión de información personal de usuarios. Se obtuvo una arquitectura basada en estándares de presentación y manejo de datos, así como implementación de mecanismos y servicios de seguridad, por lo que esta arquitectura garantiza una adecuada administración de la información, de forma que la misma se encuentre siempre disponible favoreciendo su movilidad y ubicuidad.

Palabras clave: Arquitectura, Cómputo Ubicuo, datos personales, datos sensibles, Gestión de Información, procesos organizacionales.

\section{Abstract}

Information has a fundamental role for decision-making in organizations and even daily life. People and organizations now have their information in physical form (official documents) or digital (digital photographs or pdf documents) and are managed through different protocols, applications and systems such as banks, payroll, social networks and databases to mention someone's in all cases there is some level of protection and confidentiality to ensure the integrity, consistency and completeness of the information, in addition to the existence of architectures oriented to the communication and sharing of data between systems. However, complete interoperability between them is still not achieved in a way that is possible to share information in a secure and transparent method to users.

In this article the objectives are: 1) Perform an analysis of the existing architectures, 2) describe the process followed in the design of an architecture that is proposed for the 
management of personal information, and 3) determine the functionalities of each stage. The research is descriptive in accordance with the stated objectives and for the construction of the architecture the steps of the scientific method were followed.

The results obtained are summarized in the generation of an architecture that is presented as a guide for the development and integration of systems dedicated to the management of personal information of users, not only of mobile devices to promote ubiquity, but of any type of system Computer science. An architecture based on standards of presentation and data management, as well as implementation of security mechanisms and services, was obtained, so that this architecture guarantees an adequate administration of the information, so that the information is always available favoring its mobility and ubiquity.

Key words: Architecture, Ubiquitous Computing, Personal Data, Sensitive Data, Information Management, Organizational Processes.

\section{Resumo}

Informação desempenha uma chave para a tomada de decisão nas organizações e inclusive vidas diárias. Atualmente, indivíduos e organizações têm seus dados em forma física ou digital, a ser gerido por diferentes protocolos, aplicações e sistemas, como bancos, folha de pagamento, redes sociais e bases de dados para citar alguns; em todos os casos ele tem algum nível de proteção e confidencialidade para garantir a integridade e consistência da informação, bem como a existência de arquiteturas orientadas a comunicação e partilha de dados entre sistemas. No entanto, mesmo a plena interoperabilidade entre eles, para que possível a partilha de informação forma segura e transparente aos usuários não é alcançado.

Neste artigo, os objetivos são: 1) Realizar uma análise da arquitetura existente, 2) descrever o processo de concepção de uma proposta para a arquitetura de gerenciamento de informações pessoais e, 3) para determinar a funcionalidade de cada etapa. A pesquisa é descritiva de acordo com os objectivos e etapas arquitetura do método científico é seguido.

Os resultados são resumidos na geração de uma arquitetura que é apresentado como um guia para o desenvolvimento e integração de gestão de pessoal usuários de sistemas de 
informação dedicados. foi obtida uma arquitetura baseada em padrões de apresentação e gerenciamento de dados e implementação de mecanismos e serviços de segurança, de modo que esta arquitetura garante a gestão da informação adequada, de modo que é sempre disponível mobilidade favorecendo e onipresença.

Palavras-chave: Arquitetura, Computação Ubíqua, dados pessoais, dados sensíveis, gestão da informação, processos organizacionais.

Fecha Recepción: Agosto $2016 \quad$ Fecha Aceptación: Diciembre 2016

\section{Introducción}

Diversas organizaciones se encuentran en un momento de transición debido a los avances tecnológicos que se han suscitado en los últimos años. Su propio crecimiento ha propiciado, además, cambios en los requerimientos de negocios por lo que ha sido necesario migrar sus sistemas y servicios con la finalidad de implementar sistemas más complejos. Dado que es primordial para la toma de decisiones en una organización conocer en tiempo real la información, la gestión de la misma constituye una parte clave del diseño de las aplicaciones y sistemas informáticos.

El cómputo móvil tiene como finalidad el tratamiento de información por medio de "dispositivos computacionales con capacidad de movilidad y con acceso digital a fuentes de información vía una infraestructura de comunicación" (Guzmán, 2012), por lo que se ha convertido en una gran herramienta impulsora de las organizaciones para optimizar y potenciar el manejo de la información del negocio, especialmente la que es de carácter confidencial, puesto que se tienen al alcance diversos tipos de dispositivos y aplicaciones móviles que permiten capturar, consultar y almacenar información dinámicamente desde cualquier lugar, sin necesidad de seguir atados a los estáticos centros de cómputo, de acuerdo a lo señalado por Medina, (2012). 
El presente artículo se organiza como se describe a continuación. En la primera parte, referida al contexto, se presenta una breve introducción al cómputo móvil y su integración a la vida diaria de las personas, así como las necesidades organizacionales actuales de manejo de información. Posteriormente se introduce la problemática que da lugar a la necesidad de generar procesos de gestión de información globales para posteriormente presentar los objetivos de la investigación. En el apartado de metodología se presenta el método de investigación planteando que corresponde al método científico. En una primera etapa se realiza un análisis de las estructuras existentes, se revisa lo referente a interoperabilidad entre sistemas de gestión de información, y en el rubro de resultados, se dan a conocer los procesos y funciones requeridos para cada una de las 6 Capas que corresponden a la arquitectura propuesta que se caracteriza por permitir el desarrollo e interoperabilidad entre sistemas dedicados a la gestión de información personal. Las conclusiones y direcciones futuras del trabajo se describen en el último rubro.

\section{Contexto}

En la actualidad, la información se ha convertido en una pieza fundamental de la mayoría de los procesos en diversos sectores económicos, productivos y sociales. En lo concerniente a la información personal, para su gestión y compartición se han desarrollado diversas herramientas tecnológicas que deben apegarse a legislaciones y protocolos que determinan su manejo, distribución y protección.

Con la intención de adaptarse a los cambios tecnológicos de la era digital actual, las organizaciones han realizado fuertes inversiones en aplicaciones de software para brindar soporte a sus procesos de negocio. La infraestructura que se implementa de acuerdo a los requerimientos es normalmente heterogénea a través de un número de plataformas, sistemas operativos y aplicaciones desarrolladas en diferentes lenguajes, generando regularmente redundancia y traslape en la funcionalidad y servicios, dando como resultado un desperdicio de recursos valiosos, generando tiempos de respuesta inadecuados. Los administradores de negocios y tecnologías de la información implementan arquitecturas para poder desarrollar soluciones que eliminen las desventajas citadas y sean capaces de trabajar en colaboración para compartir la información dentro de una organización. 
En aras de adaptarse a las necesidades tecnológicas y organizaciones de hoy en día, aún no se ha logrado llegar a una arquitectura estándar deseable, debido a las desventajas que presentan los esquemas arquitectónicos que enseguida se enlistan:

a. La integración de una arquitectura agrega mayor complejidad en términos de latencia de red, formato de mensajes, balanceo de carga y tolerancia a fallos.

b. Complejo manejo de meta-datos.

c. Complejo manejo de los niveles de seguridad.

d. No existen herramientas robustas para llevar a cabo pruebas.

e. Falta de confianza horizontal por parte de las organizaciones en la implementación de arquitecturas.

f. La información requiere formateo de datos aumentando tiempos de procesamiento y respuesta.

g. La consistencia de datos no es completamente segura.

\section{Problema de investigación}

Los sistemas informáticos de las organizaciones poseen al menos un área dedicada a la administración de la información, dado que ésta debe encontrarse disponible en todo momento. Las organizaciones han ido adaptando su infraestructura organizacional a la integración de soluciones móviles (Medina, 2012). Sin embargo, existe aún un enorme vacío sobre las posibilidades de utilización de la computación móvil para el manejo de información personal confidencial, por lo que se propone el diseño una Arquitectura de Gestión de Información Personal que permita compartir información entre sistemas organizacionales, a través de diferentes dispositivos, sin importar sus características de infraestructura. 


\section{Objetivos}

1. Realizar un análisis de las arquitecturas existentes.

2. Describir proceso seguido en el diseño de una arquitectura que se propone para la gestión de información personal.

3. Definir las funcionalidades de cada Capa que integra la arquitectura propuesta.

\section{Hipótesis}

El modelo de capas para la construcción de la arquitectura permite que se dé la interoperabilidad entre sistemas.

\section{Metodología}

En este apartado se describe el proceso seguido para alcanzar los objetivos propuestos y con ello dar solución a la problemática planteada, para lo cual la investigación se apoyó en el método científico.

En la primera etapa se trabaja lo correspondiente a lo señalado en el primer objetivo, ya que se hace un análisis de las arquitecturas existentes, y de acuerdo a las características encontradas se realiza una primera clasificación; se continúa con una segunda clasificación, pero ésta considera sólo a las arquitecturas centradas en procesos. En esta etapa también se revisan las características de manejo de información, abordadas por los sistemas de información personal mayormente implementados en la actualidad.

Con la segunda etapa del método, se busca alcanzar el segundo objetivo, por lo que se plantea el diseño de la arquitectura mediante la construcción de 3 Capas. Para finalizar esta parte de la metodología se determinan los flujos de trabajo concerniente a cada Capa.

\section{Etapa 1. Análisis de arquitecturas existentes}

En la búsqueda de hacer más eficientes los procesos de manejo de información e interoperabilidad entre sistemas, se han llevado a cabo desarrollos bajo diversas arquitecturas, según las posibilidades de infraestructura así como herramientas de hardware y software con que se contaba. En la Fig. 1 se muestra la evolución que han tenido las 
arquitecturas en sistemas computacionales, que corresponde con avances en hardware y comunicaciones.

Figura 1. Evolución de arquitecturas computacionales.

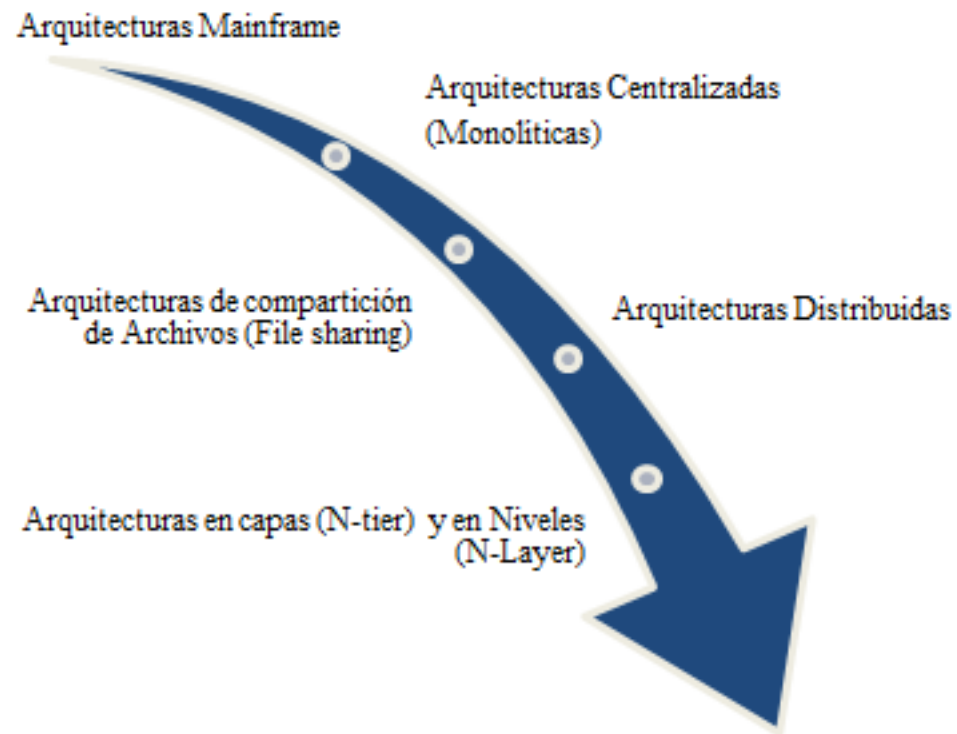

En la Tabla 1 se resumen los aspectos más relevantes de cada una de ellas. 
Tabla 1. Características de Arquitecturas

\begin{tabular}{|c|c|}
\hline Arquitectura & Características \\
\hline Mainframe & $\begin{array}{l}\text { Procesos centralizados. } \\
\text { Dependencia total del Mainframe para llevar a cabo los } \\
\text { procesos. } \\
\text { Terminales brutas (No poseen CPU). } \\
\text { No soporta GUI. } \\
\text { No es Cliente/Servidor. }\end{array}$ \\
\hline Centralizada & $\begin{array}{l}\text { Monolítica (Consta de una máquina, un proceso y un } \\
\text { usuario). } \\
\text { No existe ninguna posibilidad de concurrencia. } \\
\text { Requiere de dispositivos externos para compartir } \\
\text { información. }\end{array}$ \\
\hline $\begin{array}{l}\text { Compartición de Archivos } \\
\text { (File-sharing) }\end{array}$ & $\begin{array}{l}\text { Una máquina comparte recursos (archivos) y una terminal } \\
\text { realiza peticiones sobre los recursos y trabaja sobre ellos. } \\
\text { Los procesos se realizan en la terminal que solicitó el } \\
\text { servicio. } \\
\text { Terminales inteligentes. } \\
\text { Soporta GUI. } \\
\text { Bajo nivel de tráfico, archivos pequeños, pocas } \\
\text { actualizaciones. } \\
\text { Bajo nivel de concurrencia (12 usuarios máximo) }\end{array}$ \\
\hline Distribuida & $\begin{array}{l}\text { Permite implantar sistemas colaborativos en la web. } \\
\text { Mayor aprovechamiento del hardware y ancho de banda. } \\
\text { Mayor seguridad y autonomía. } \\
\text { Manejo de concurrencia. } \\
\text { Permiten que los clientes trabajen GUI mientras el servidor } \\
\text { se centra en otros procesos. }\end{array}$ \\
\hline En Capas & $\begin{array}{l}\text { Separación en componentes dedicados a una labor en } \\
\text { particular favoreciendo la distribución de procesos y roles. }\end{array}$ \\
\hline
\end{tabular}

Debido al gran auge de aplicaciones para internet, la arquitectura en Capas (Zimmerman, 1999 y Mora, 2011) ha sido ampliamente implementada. Sin embargo, para mantenerse competitivas, las organizaciones deben ser ágiles en la adaptación de sus procesos de negocio, así como a los cambios que se generan en el entorno, por lo tanto, cada vez más se han centrado en procesos, lo que significa que las actividades que llevan a cabo las organizaciones están siendo estructuradas como procesos. Dada esta situación, las organizaciones han desarrollado una variedad de estrategias para entender y formalizar sus 
procesos, así como mantener su documentación disponible y al día, con la tendencia a diseñar componentes para uso general, tomando en cuenta sus propiedades de ser reutilizados y compartidos entre múltiples aplicaciones.

\section{Arquitecturas centradas en procesos}

En la Tabla 2 se muestra un cuadro comparativo acerca de las características de las arquitecturas basadas en procesos para los sistemas organizacionales actuales.

Tabla 2. Cuadro comparativo de características de arquitecturas centradas en procesos.

\begin{tabular}{|c|c|c|c|c|c|}
\hline Arquitectura & EIA & BPA & EDA & PA & SOA \\
\hline Gestión de procesos de negocio & & & & & \\
\hline $\begin{array}{l}\text { Reingeniería de procesos de } \\
\text { negocio }\end{array}$ & $>$ & & & & \\
\hline Interoperabilidad & 7 & & & & \\
\hline $\begin{array}{l}\text { Reutilización de componentes de } \\
\text { software }\end{array}$ & $>$ & & & & \\
\hline $\begin{array}{l}\text { Portabilidad (Independencia de } \\
\text { software y plataforma) }\end{array}$ & X & \rangle & $X$ & , & \\
\hline \multicolumn{6}{|l|}{$\begin{array}{l}\text { Procesos centrados en la Gestión } \\
\text { de la Información }\end{array}$} \\
\hline $\begin{array}{l}\text { Procesos centrados en el } \\
\text { cómputo ubicuo }\end{array}$ & & & & & \\
\hline
\end{tabular}
Sturm,2015), PA (Moreno, 2015), SOA (Pesantes, Mitre y Mejía 2012).

\section{Sistemas de Gestión de Información Personal}

Desde que se adoptaron los sistemas informáticos para facilitar la gestión de información en las organizaciones, los Sistemas de Gestión de Información Personal (Personal Information Management Systems - PIMS), han constituido un elemento básico de todo sistema organizacional (Ruan, Zhang, Hua, 2010). 
Los PIMS se han desarrollado de acuerdo al tipo de información que manejan, por ejemplo, Rustom, Nasar, Mohd y Ali (2011a y 2011b), han ahondado en el estudio de interfaces de usuario en los PIMS y sus características para permitir a los usuarios interactuar con su información personal. Por otra parte, en un artículo publicado por los mismos autores (Rustom, Nasar, Mohd, Ali, 2011 y 2011b) se hace referencia al manejo de imágenes fotográficas y video a partir del estudio del ciclo de vida de un sistema PIM, mismo que resalta características como ubicuidad y gestión de la información.

En el ámbito empresarial, las soluciones móviles empresariales PIM, se utilizan para mejorar la gestión de la información personal de los empleados. Según Kamesh (2011), las PIM son aquellas soluciones que permiten incrementar la productividad personal de los empleados mediante la utilización de dispositivos móviles que corren aplicaciones para el manejo de la información personal como calendarios, administradores de contactos, programadores de eventos, alarmas, correo electrónico, etc.

En la Tabla 3 se muestra un cuadro comparativo sobre las características de manejo de información abordadas por los sistemas de información personal mayormente implementados en la actualidad. 
Tabla 3. Cuadro comparativo de características de PIMS.

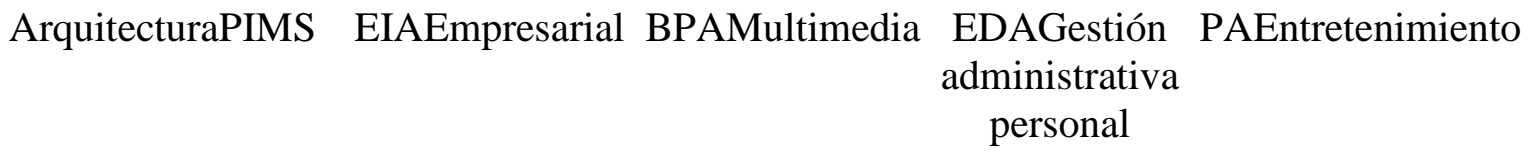

Gestión de procesos de negocio

X X X

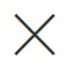

Reingeniería de procesos de negocio
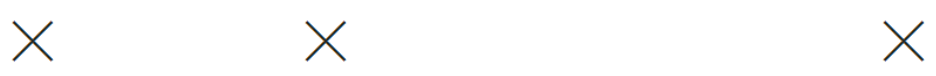

Interoperabilidad
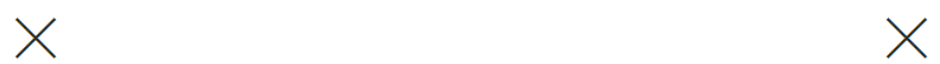

Reutilización de componentes de software
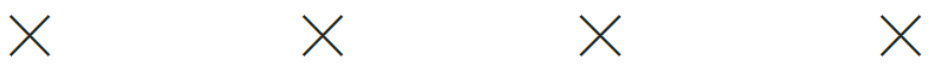

Portabilidad (Independencia de software y plataforma)
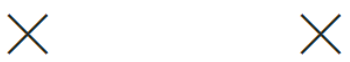

$X$

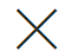

Procesos centrados en la Gestión de la Información
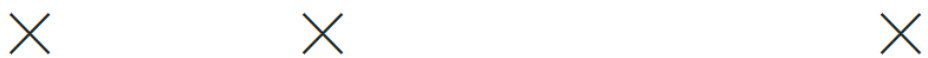

Procesos centrados en el cómputo ubicuo

\section{Etapa 2. Diseño}

El diseño arquitectónico presentado se centra en la información. El núcleo de información de interés es el correspondiente a la trayectoria personal de un individuo, misma que se caracteriza por medio de la unidad $u$ objeto inmerso en una serie de elementos computacionales que permitan portarlo y en general, administrarlo en todo momento (disponibilidad) y en todo lugar (ubicuidad). La estructura del objeto se muestra en la Figura 2: 
Figura 2. Estructura básica de Objeto Personal.

\begin{tabular}{|c|}
\hline ObjetoPersonal \\
\hline Características \\
Curp \\
Nombre \\
Apellido paterno \\
Apellido materno \\
Trayectoria Académica \\
Trayectoria Laboral \\
\hline Acciones \\
compartir() \\
validar() \\
actualizar() \\
\hline
\end{tabular}

CURP (Clave Única de Registro de Población).

Este objeto es la base de un diseño más amplio de elementos que componen la trayectoria de una persona. Por otra parte, dicho objeto se encuentra inmerso en un ecosistema digital (Kamesh, 2012), donde se deben proveer los medios necesarios para portarlo (dispositivo móvil) y dotarlo de las capacidades de ser compartido (interoperabilidad).

El diseño arquitectónico contempla que en el ecosistema cada elemento convive con otros que permitirán administrar la información de manera coherente, eficaz y segura. Para tal propósito, la arquitectura posee una distribución lógica de los procesos de negocio de tipo nlayer, con la finalidad de asegurar la separación entre la lógica de negocios y la lógica de diseño, de esta forma cada Capa interactúa con sus Capas adyacentes, lo que permite aislar las funcionalidades de cada una de ellas y obtener productos de las funcionalidades de las Capas superiores e inferiores.

El estilo establecido para la arquitectura es bottom-up (ascendente), donde cada elemento de una Capa $i$ notifica a elementos de la Capa superior $i+1$ de que ha generado algún producto que le es de interés. La Figura 3 muestra la arquitectura en 6 Capas. Cada Capa (i) es 
independiente e interactúa con la Capa siguiente (i+1) proporcionándole elementos productos de su funcionalidad.

Figura 3. Arquitectura de Gestión de Información Personal en un entorno Móvil.

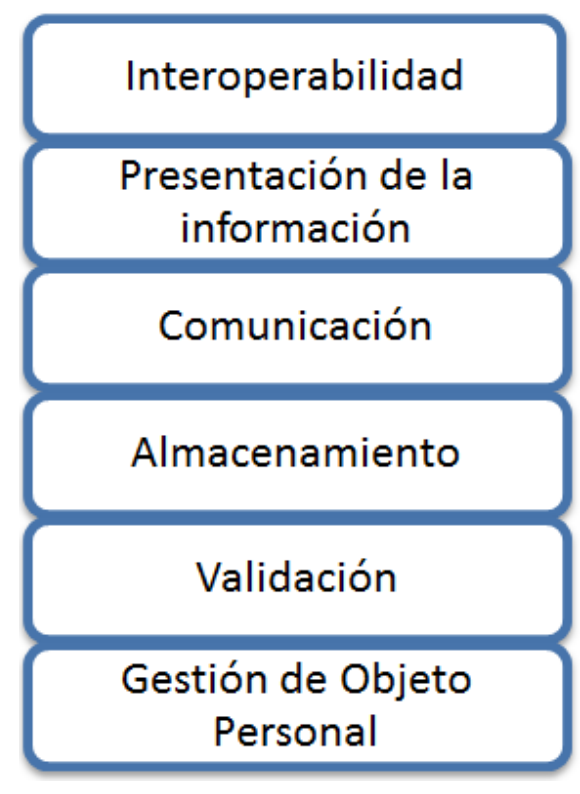

\section{Etapa 3. Funcionalidad de las Capas y sus flujos de trabajo}

\section{Descripción de la funcionalidad de cada Capa}

Todo sistema de información basado en la arquitectura, debe contemplar como precondición la preparación de la información a fin de disponer de la misma en un formato digital estándar. A continuación se describen las funciones mínimas requeridas:

Precondición: Preparación de la información

a. Obtención. Se debe llevar a cabo la obtención de la información que integra el perfil personal de una persona (datos personales, datos sensibles, trayectoria profesional, trayectoria educativa) disponible en formatos heterogéneos.

b. Verificación. Revisión de la información por parte de una entidad de confianza con la finalidad de verificar su procedencia y contenido. 
c. Digitalización. El mismo propietario o una entidad capacitada procederá a la edición de información en formato digital estándar Multipurpose Internet Mail Extensions (MIME).

Dadas las condiciones establecidas en la precondición, la funcionalidad de cada Capa se describe a continuación:

1. Gestión de objeto personal.

d. Recepción. Aceptación de la información que integra el perfil personal de una persona (datos personales, datos sensibles, trayectoria profesional, trayectoria educativa) en archivos con formato digital estándar.

e. Gestión de Objeto. Se crea y/o edita el objeto personal del propietario de la información.

2. Validación.

f. Certificación de entidades. Establecimiento de una entidad de confianza global que certifique y a su vez otorgue habilidad a sus entidades certificadas parciales para validar la información.

g. Certificación de propietario. Generación de Certificado Digital firmado digitalmente por entidad de confianza y par de llaves (pública y privada) de autentificación para el propietario del objeto personal.

h. Firmado Digital de Objeto Personal. Certificación de la validez de la información por parte de una o más entidades de confianza certificadas, es decir, cada documento que integra el perfil personal debe firmarse digitalmente por alguna de dichas entidades.

3. Almacenamiento.

i. Interfaz de gestión. Generación de interfaz de software denominada Gestor de Objetos Personales encargada del almacenamiento y 
administración de los mismos así como de Certificado Digital y par de llaves de seguridad (pública y privada) en dispositivo móvil.

j. Repositorio de Objetos. Gestión de una unidad de almacenamiento de todos los objetos personales validados.

4. Comunicación.

k. Distribución. Medio o servicio para colocar el objeto personal a disposición de otros sistemas.

5. Presentación de la información.

1. Formato. Presentación de datos sujeta a estándares de formato de datos.

6. Interoperabilidad.

m. Colaboración con otros sistemas. Brindar la cualidad de compartir e intercambiar información con otros sistemas de forma transparente al propietario.

n. Filtrado. Establecimiento de niveles de compartición de información según preferencias del propietario, solo transfiere lo que el usuario desea.

La forma en que colabora cada Capa con las adyacentes dentro del modelo, se muestra en la Figura 4, en donde cada Capa contiene procesos propios que genera entregables para la respectiva Capa superior. 
Figura 4. Funciones para cada Capa de la arquitectura.

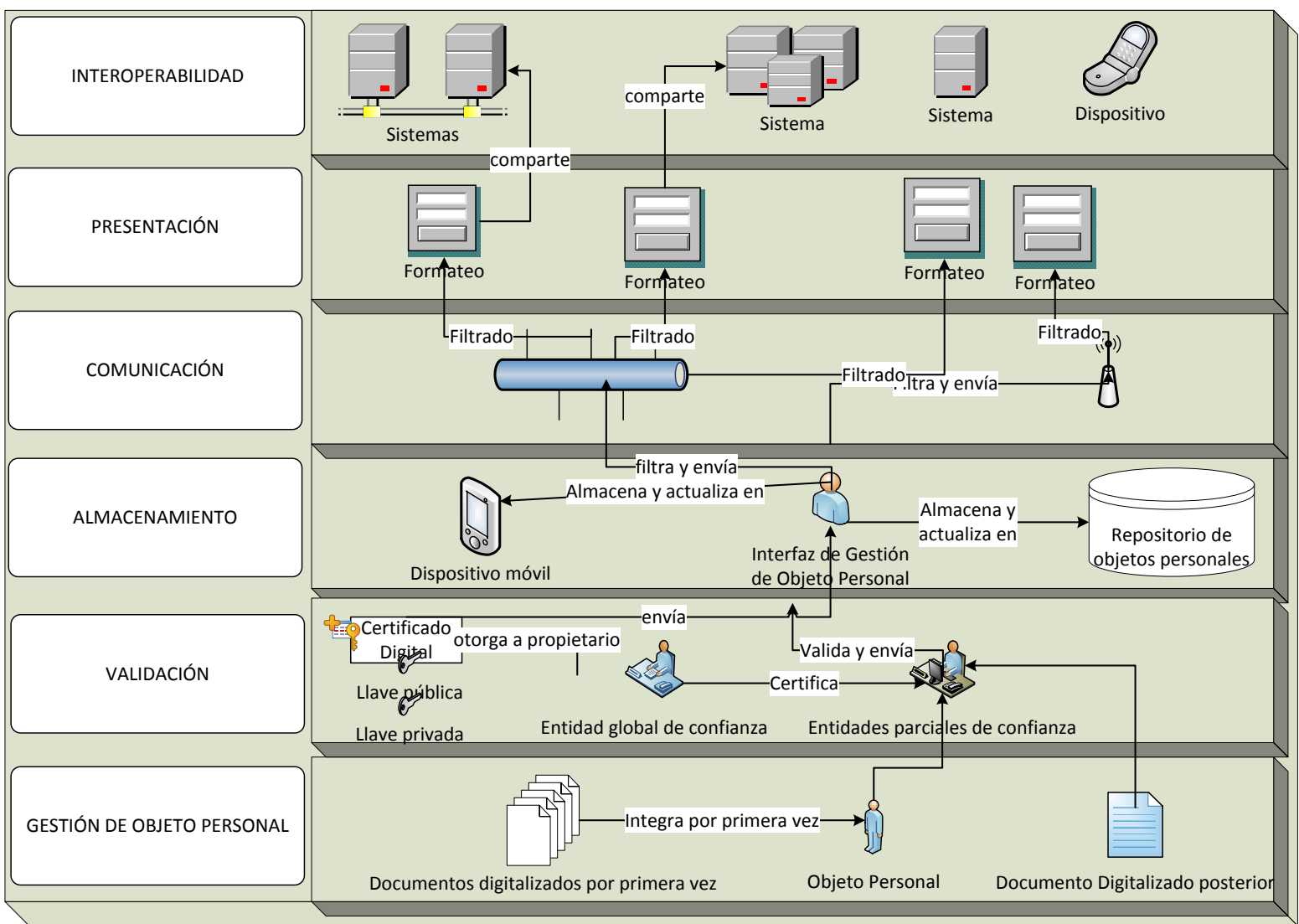

Flujos de trabajo de cada Capa

Previo a definir los procesos correspondientes a las Capas de la arquitectura, se exponen las actividades que deben llevarse a cabo según se estableció anteriormente como precondición (preparación de la información). Esta etapa es relevante debido a que provee a la primera Capa de la arquitectura con los insumos necesarios para su adecuado tratamiento posterior.

1. Preparación de la información.

En el proceso de preparación de la información resalta la existencia de la entidad encargada de la verificación de información, dicha entidad debe poseer herramientas y soporte adecuado para contar con la capacidad de determinar la originalidad de los documentos e información. En la Figura 5 se muestra el proceso por medio del cual los documentos son verificados, aceptados y digitalizados según las funcionalidades descritas como precondición. 
Figura 5. Proceso de preparación de la información (precondición).

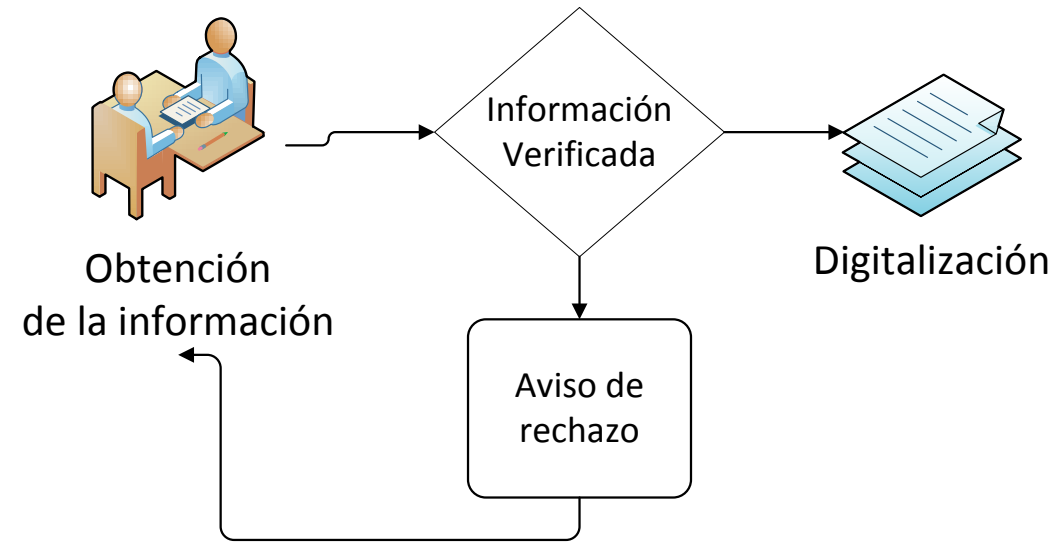

Una vez cumplidas las condiciones adecuadas, producto de la etapa de preparación de la información, se procede a describir cada proceso correspondiente a las Capas que integran la arquitectura.

\section{Capa 1.}

En la Figura 6 se ilustra el proceso en la Capa 1 donde una vez que se dispone de la información personal en formato digital, se procede a tomar una de las siguientes decisiones:

o. En caso de que el propietario no disponga de objeto personal, éste se gestiona por primera vez y se procede a enviarlo a la Capa 2 para su validación mediante cada entidad de confianza correspondiente.

p. En caso contrario, es decir que ya se disponga de objeto personal, los documentos digitalizados se envían a la Capa 2 para su validación por cada entidad de confianza correspondiente. 
Figura 6. Proceso de gestión de objeto personal en Capa 1.

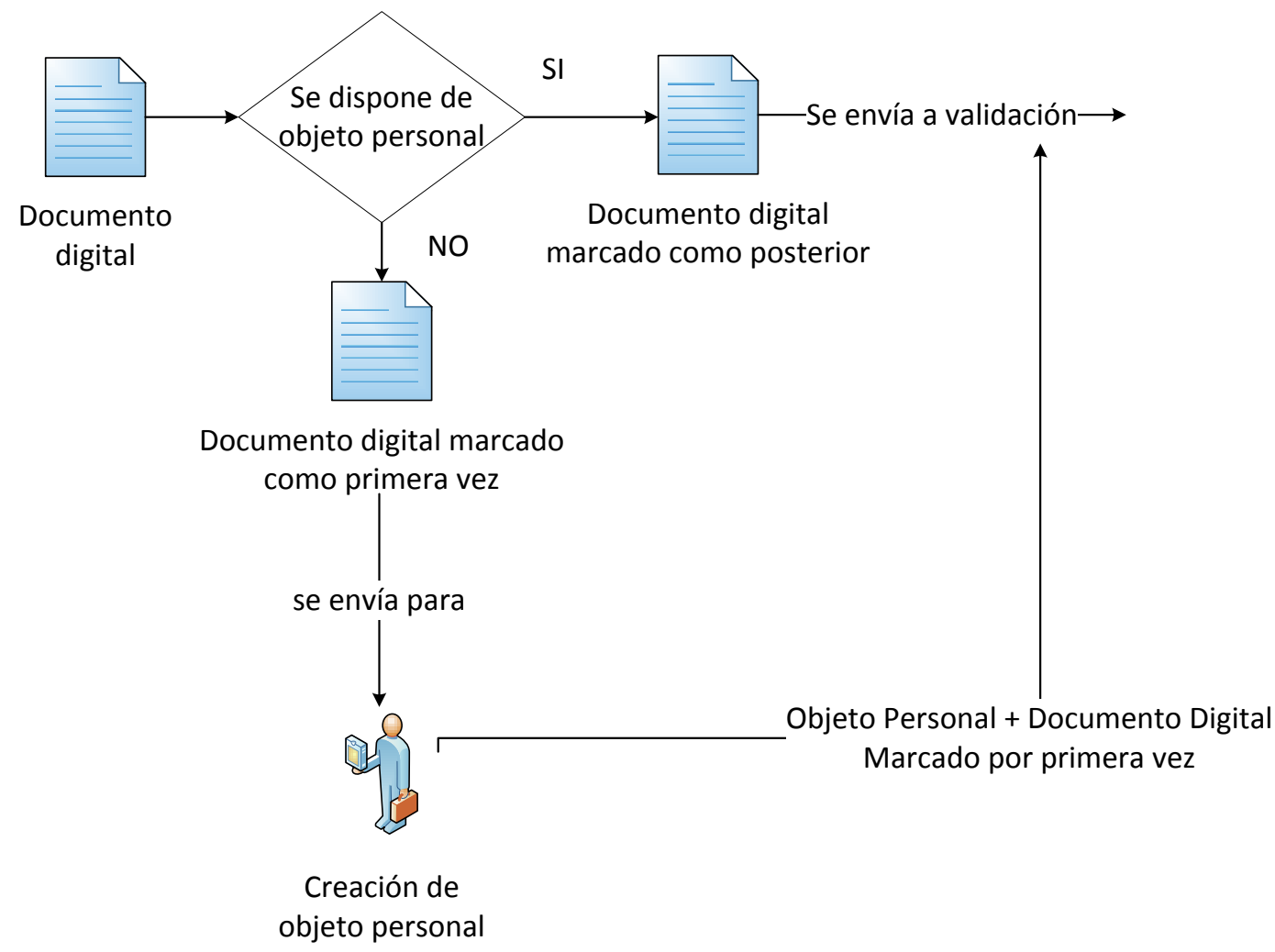

3. Capa 2.

El esquema arquitectónico debe encontrarse operando en un entorno de confianza, por lo que la certificación de los documentos radica en el establecimiento de una entidad global de confianza, encargada de certificar a todas las entidades participantes dotándolas de la capacidad de validar información. Esta entidad es también quien otorga certificado digital y llaves a los propietarios con la finalidad de proporcionarles la capacidad de administrar su propia información. Figura 7. 
Figura 7. Proceso de Certificación de entidades participantes en Capa 2.
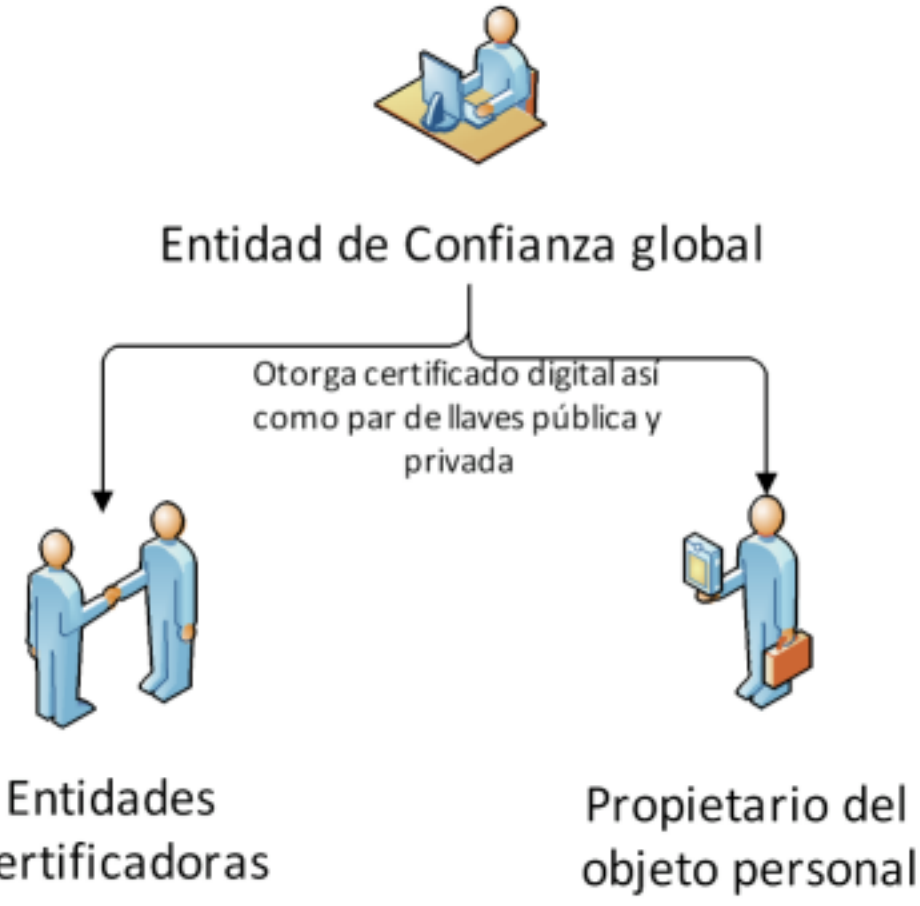

De esta forma cada entidad certificadora valida la información verificada firmándola digitalmente según el proceso en Figura 8.

Figura 8. Proceso validación de documento en Capa 2.

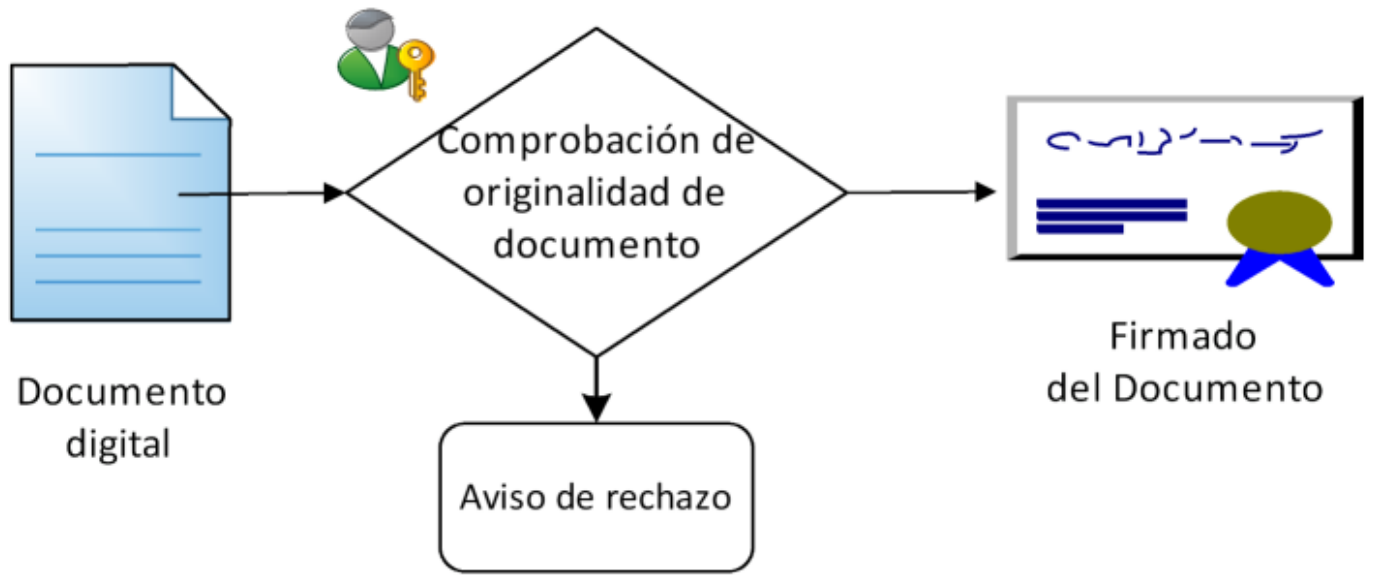


4. Capa 3.

En esta etapa se requiere de una interfaz para el almacenamiento, distribución y transporte tanto del objeto personal como del certificado digital y par de llaves del propietario en un dispositivo móvil. Por otra parte, la interfaz de gestión de objeto personal debe ocuparse del proceso necesario para anexar los documentos firmados digitalmente al objeto personal de manera que siempre esté actualizado. Es también responsable del almacenamiento o actualización en un repositorio de objetos (Figura 9), para permanecer a disposición de su propietario.

Figura 9. Proceso de almacenamiento y actualización en Capa 3.
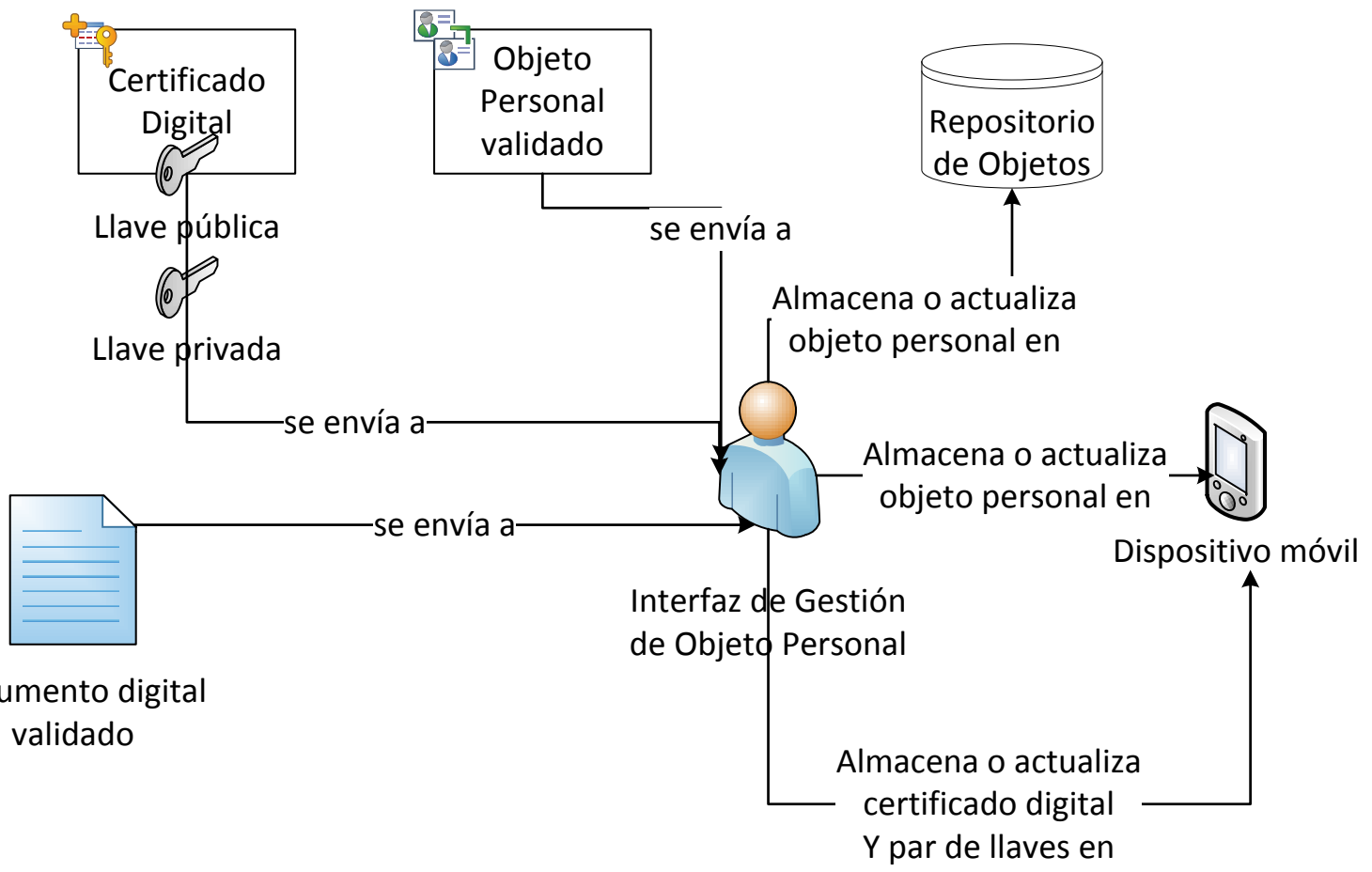

5. Capa 4.

El objeto personal ahora debe ahora ponerse a disposición de los demás sistemas, a través de cualquier medio o servicio de comunicación, asegurando su confidencialidad, integridad y disponibilidad (Figura10). 
Figura 10. Disponibilidad del objeto personal en Capa 4.

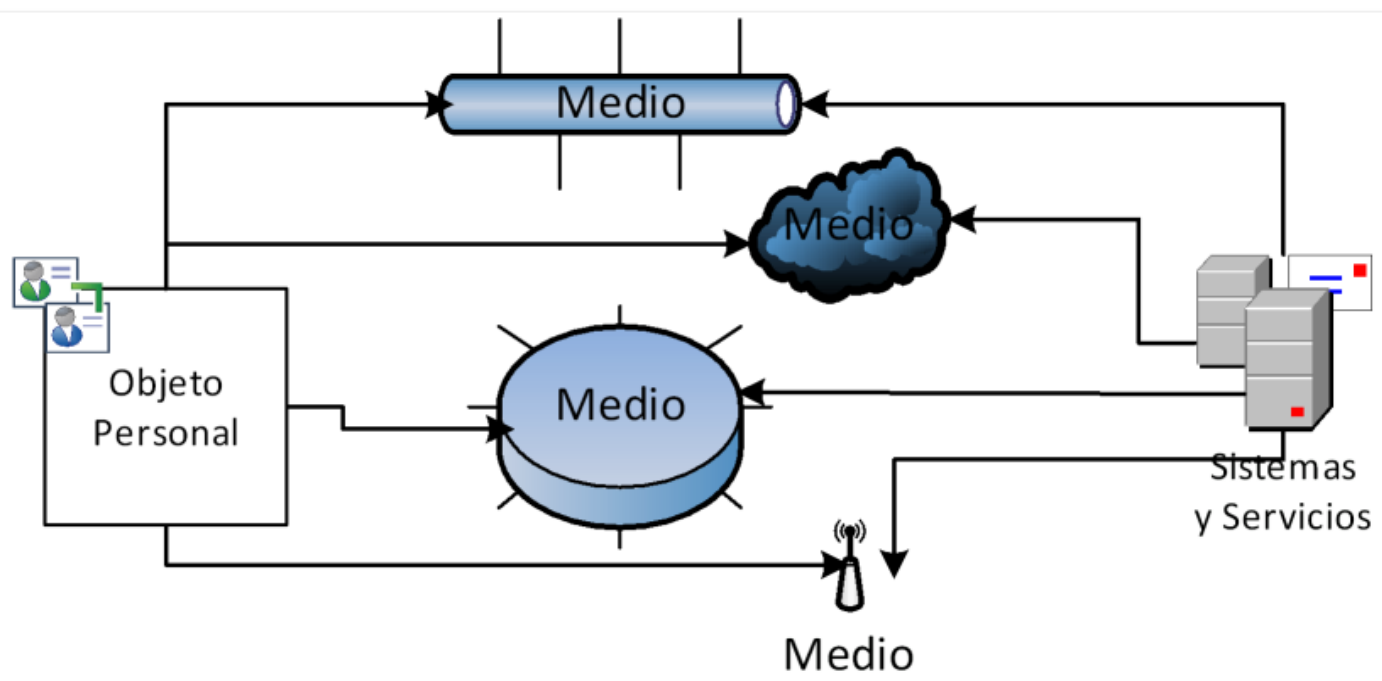

6. Capa 5.

Para compartir la información mediante el objeto personal, es requerimiento básico que los documentos se encuentren en formatos MIME bajo estándares de presentación de datos con la finalidad de otorgar la capacidad de adaptabilidad a diversos sistemas de información.

Por otra parte, cabe mencionar que no todo el contenido debe estar disponible para todos los sistemas, sino sujeto a las preferencias del propietario, por lo que él mismo debe tener la capacidad de seleccionar de manera conveniente por medio de la implementación de un servicio de filtrado, la información que desea compartir. El servicio filtrado no deberá sobrepasar en ningún caso su alcance, es decir, la información del objeto personal a la que tenga acceso cualquier sistema o servicio debe ser única y exclusivamente aquella que el propietario desee proporcionar no existiendo algún medio o acción que viole esta premisa según se aprecia en la Figura 11. 
Figura 11. Formato de contenido del objeto personal en Capa 5.

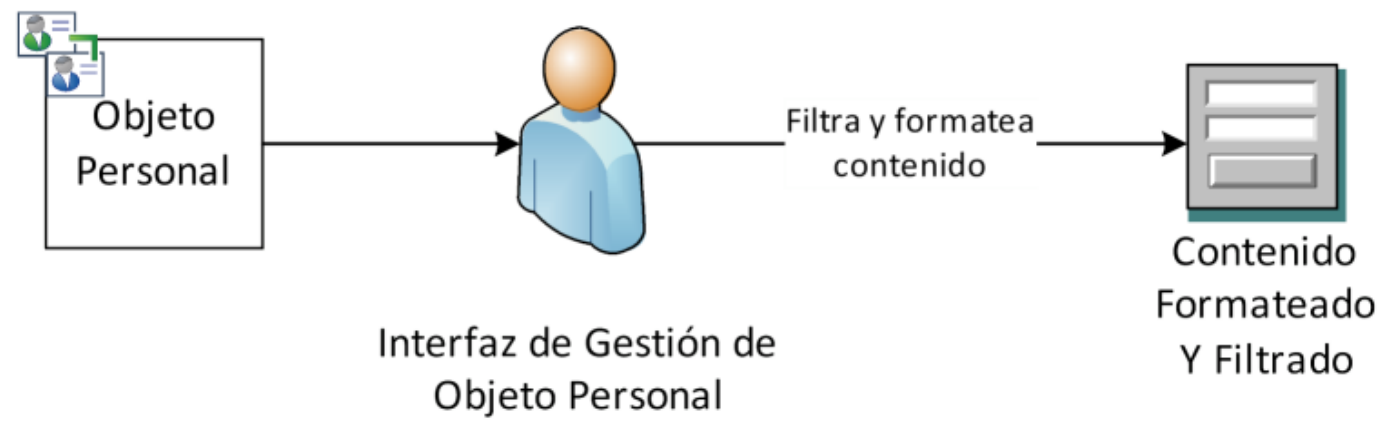

7. Capa 6.

La interoperabilidad e integración de sistemas son tópicos de necesidad prioritaria en esta arquitectura, puesto que la colaboración con otros sistemas y servicios asegurará la disponibilidad de la información. Esta es la única Capa que tendrá comunicación con otros sistemas. La Figura 12 ilustra el mecanismo de compartición de información e interoperabilidad con otros sistemas.

Figura 12. Compartición de la información para la integración con otros sistemas

Capa 6.

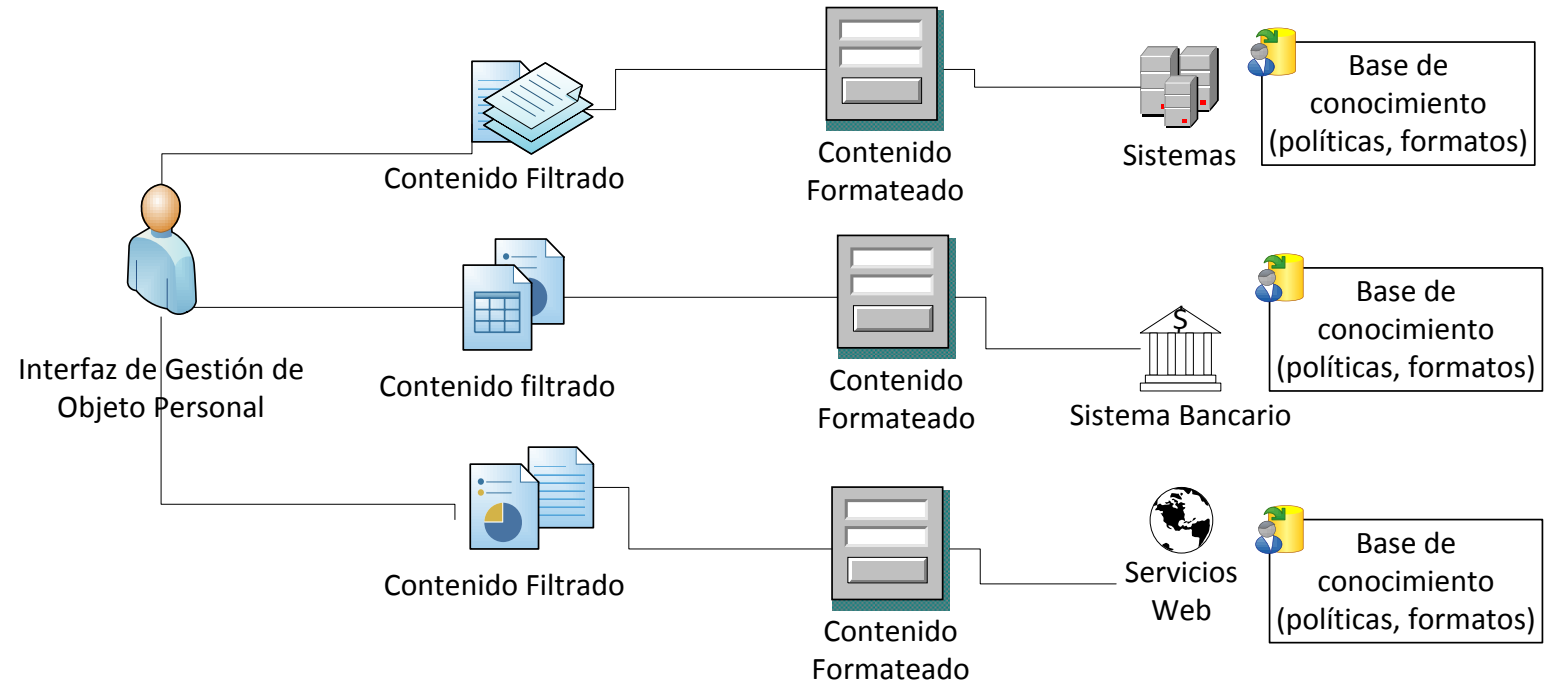


Se emplearon modelos de Casos de Uso para representar una vista de la arquitectura, mostrándose aquí el correspondiente Gestor de Objeto Personal, quien es el actor que tiene una mayor interacción en el diseño (Figura 13).

Figura 13. Modelo de Casos de Uso del gestor de objeto personal.

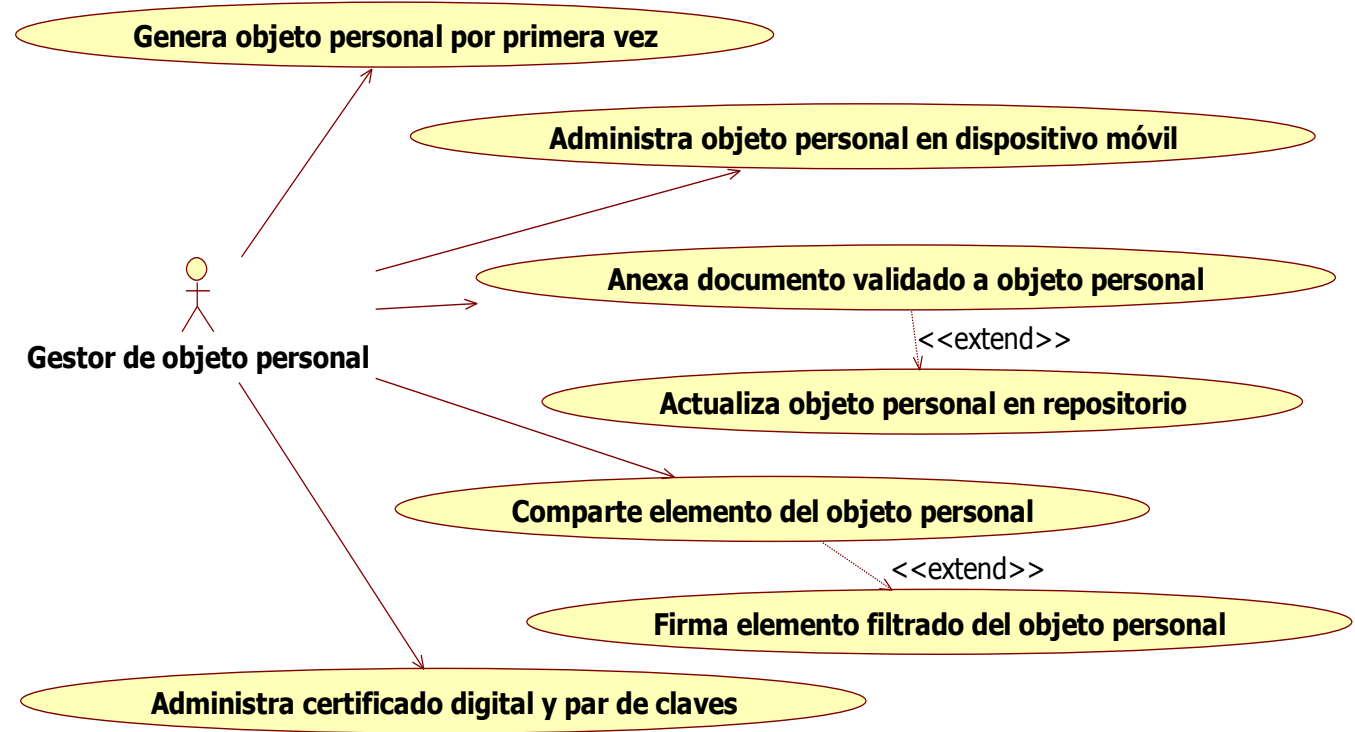

\section{Resultado}

La arquitectura, de la cual se describió su proceso de construcción, se encuentra diseñada bajo los principios de:

a. Escalabilidad. Por su capacidad para adaptarse a los procesos y lógica de negocio.

b. Empleabilidad. Por el potencial que posee de ser aplicada en diversas organizaciones.

c. Interoperabilidad. Gracias a su habilidad para interactuar y compartir información por medio de interfaces de comunicación estandarizadas con otros sistemas existentes sin restricción alguna. 
d. Portabilidad. Asegurando una dependencia nula del software en que se desarrollen los procesos.

Los principios mencionados dotan a la arquitectura con las siguientes características:

a. Ser Estructurada, por corresponder a un diseño estratificado y modular.

b. Ser Flexible en sus procesos ante cualquier situación organizacional, basada en estándares con la finalidad de asegurar la integración con otros sistemas y adaptable a los procesos y lógicas de negocio de las organizaciones.

Dentro de un entorno organizacional, la arquitectura define la estructura, organización y la relación, entre lógica de negocio y tecnología del mismo, encontrándose constituida por procesos representados por modelos para lograr integración y coordinación entre los sistemas. Se compone además de un conjunto de procesos de negocio modulares reconfigurables con alta cohesión y descripciones basadas en protocolos e interfaces. Soporta asimismo componentes y servicios débilmente acoplados para posibilitar la flexibilidad en el negocio de una manera interoperable e independiente de la tecnología. Es iterativa puesto que un proceso está compuesto de otros más particulares, por ejemplo aquellos que describen utilidades técnicas como seguridad y autenticación. Sus elementos de gestión se clasifican en 3 grupos principales mostrados en la Figura 14.

Figura 14. Elementos de la arquitectura.

- Reducción de riesgos y costos operacionales y de mantenimiento

- Reducción de rechazos e incidencias

- Mejora de los procesos

- Mejora en eficiencia

- Rentabilidad

- Adaptación

organizacional

Principios

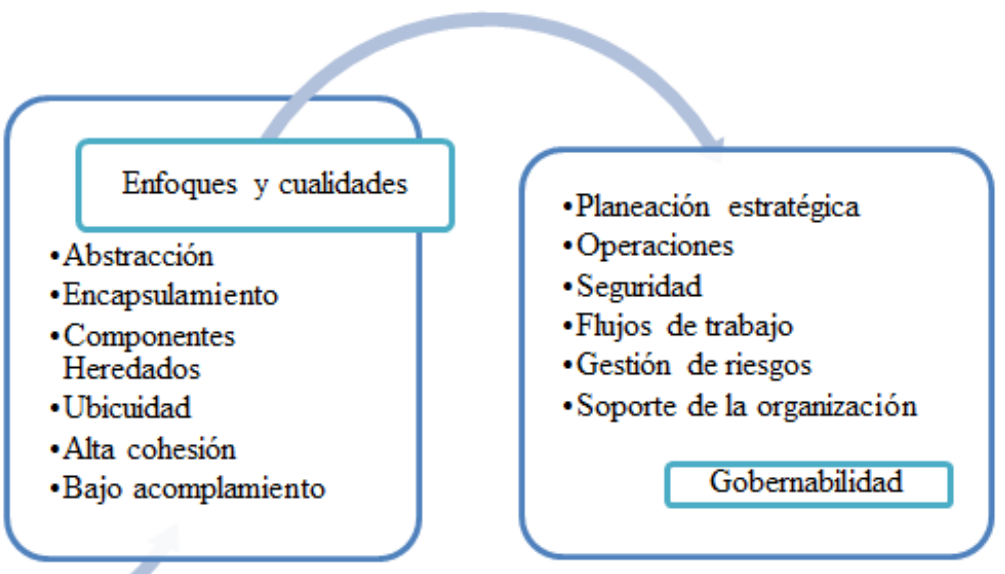


Para que se dé la interoperabilidad entre los sistemas que se implementen en la arquitectura es necesario basarse en estándares abiertos internacionales de seguridad y de intercambio de datos (Cadena, 2010). Para el caso de México se adoptarían los que están listados en la firma electrónica (SAT, 2015). Se propuso la Capa 6 ya que permite que se dé la interoperabilidad entre sistemas desarrollados siguiendo los lineamientos establecidos por la arquitectura, con lo cual se cumple la hipótesis de la investigación.

\section{Conclusiones}

El gran auge que poseen actualmente tópicos como la movilidad y ubicuidad, así como la heterogeneidad de los dispositivos móviles y sistemas, da lugar a nuevos escenarios que favorecen la cooperación entre los individuos mediante el uso generalizado de todos estos avances en materia de tecnología. En este sentido, la información como elemento básico otorga un valor añadido a dichos escenarios e implica nuevas necesidades y requerimientos para los desarrollos de sistemas organizacionales, tanto los que ya están implementados como los futuros. A partir del presente trabajo se abre una enorme posibilidad de investigación sobre la computación móvil centralizada en la gestión de información, para realizar a futuro sistemas y esquemas particularizados sobre la utilización de soluciones móviles con el fin de otorgar mayor valor a las organizaciones. 


\section{Bibliografía}

Ahmad M, Odeh M (2012). Semantic Derivation of Enterprise Information Architecture from Business Process Architecture. IEEE 79-84, ICCTA. Alexandria Egypt.

Brancheau J. C. y Wetherbe, J. C (1986). Information architectures: methods and practice," Informaton. Processing Management, vol. 22, no. 6,453-463.

Cadena Sandoval C.A. (2010). Sistema Integral de Gestión Registral de Secretaria de Economía Gobierno de la Ciudad de México, Sitio Web: http://www.firmadigital.gob.mx/ap3/anexo2.doc, México.

Guzmán Ríos R. A., (2012). Arquitectura de Servicios Web Semánticos Sensible al Contexto para Dispositivos Móviles. Tesis de maestría CINVESTAV. IPN. México.

Instituto Federal de Acceso a la Información, IFAI, (Último acceso: septiembre de 2016). http://inicio.ifai.org.mx/SitePages/ifai.aspx

Kamesh, P. (2012). The What, Why, Who and How of Enterprise Mobility Adoption. Sand Hill Group (Último acceso: junio de 2016) http://sandhill.com/article/the-what-whywho-and-how-of-enterprise-mobilityadoption.

Lapouchnian A, Yu E, Sturm A. (2015). Re-Designing Process Architectures Towards a Framework of Design Dimensions. 978-1-4673-6630-4/15, IEEE

Medina Vásquez T. (2012). Análisis de la utilización de la Computación Móvil en diferentes procesos y actividades empresariales. Universidad EAFIT, Medellín. Colombia.

Miranda, M. A. (2002). Aplicación cliente/servidor de 2 niveles utilizando DCOM/COM para gestionar remota y localmente Tablas dbf. Tesis de Maestría. Universidad de Colima. México. 
Mora, J. T. (2011). Arquitectura de software para aplicaciones Web. Tesis de Maestría. Centro de Investigación y de Estudios Avanzados del Instituto Politécnico Nacional Unidad Zacatenco. México.

Moreno S. (2015) Estudio de Arquitecturas de Software para servicios de Internet de las Cosas.. Trabajo final de grado. Escuela Técnica Superior de Ingenieros de Telecomunicación, Universidad Politécnica de Madrid. España.

Pesantes M, Lemus C, Mitre H. A y Mejía J. (2012). Software Process Architecture: Roadmap, Ninth Electronics, Robotics and Automotive Mechanics. Conference IEEE . 111-116.

Ruan D, Zhang B, Hua Yu (2010). The Realization of Shopping Sites based on SOA Architecture. International Conference on Computer Application and System Modeling ICCASM.

Rustom M, Nasar Al, Mohd M, Ali M. (2011a). A Conceptual Framework for an Interactive Personal Information Management System. International Conference on User Science and Engineering 100-105, (i-USEr).

Rustom M, Nasar Al, Mohd M, Ali M. (2011b). Personal Information Management Systems and Interfaces : An Overview. International Conference on Semantic Technology and Information Retrieval. 197-202, 28-29, Putrajaya, Malaysia.

Servicio de Administración Tributaria SAT (2015), La evolución de la Firma Electrónica SHCP Expo Feria 2015. México.

Zimmerman, James B. (1999). University of Maryland European Division - Bowie State. "Mobile Computing: Characteristics, Business Benefits, and the Mobile Framework". (Último acceso: junio de 2016) Sitio web: http://www.idi.ntnu.no/grupper/su/mowahs/links/MobileFramework.pdf 